\title{
Self healing of healthy condition at cellular level
}

\author{
Evgeniy Bryndin ${ }^{1 *}$ and Irina Bryndina ${ }^{2}$ \\ ${ }^{1}$ Research Department, Research center "NATURE INFORMATIC”, Novosibirsk, Russia \\ ${ }^{2}$ Research Department, Technological platform "FUTURE MEDICINE”, Novosibirsk, Russia
}

\begin{abstract}
The main processes of self-restoration of organism at the biological level are hormonal balance, metabolism and regeneration. For normalization of metabolism it is necessary to pass to alkaline food, having excluded from a food allowance cancerogenic, received by chemical processing, the food containing mutational proteins; to drink clear water. At normal metabolism hormonal balance requires coordination of activity with a natural daily cycle. When the natural rhythm of a dream and wakefulness is broken, then hormone melatonin is produced insufficiently, the hormonal balance in an organism is broken and, as a result, the mechanism of cellular updating is broken, and cages continue to grow or regenerate. That process of dying off of the regenerated cages has begun it is necessary to suspend their mutational genes genetic inductors (hormones). It is reached by hormonal balance on production of hormones and normalization of a hormonal background on delivery to their cages. For establishment of hormonal balance production of hormone of melatonin epifizy in enough is necessary. The condition of an organism depends on a qualitative condition of cages. By their high-quality improvement through system of cellular self-restoration of an organism the healthy condition of cages at the biological level by charging, gymnastics and physical culture, the schedule operating time, rest, acceptance of food and a dream, control of vital systems, healthy food, pure ecology, coordination of activity with a natural daily cycle, psychophysiological normalization by useful habits of healthy lifestyle is reached.
\end{abstract}

\section{Introduction}

The human body is formed by cells of different types. Their numbers range from thirty to one hundred trillion. Each cell has its own life expectancy. After the expiration of her term, the cell dies. This death is ordered and genetically programmed. In order to maintain and preserve the body 's homeostasis, there are strict systems for regulating metabolic processes. This regulation occurs at the organizational, cellular, and molecular levels.

Homeostasis has certain boundaries. When staying, especially longterm, an organism in conditions that are significantly different from those to which it is adapted, homeostasis is disturbed, and shifts that are incompatible with normal life can occur. Thus, with a significant change in the external temperature to the side of both its rise and decrease, the body temperature may increase or decrease accordingly, and overheating or cooling of the body resulting in death may occur. With significant restriction of water and salts ingestion into the body or complete deprivation of its substances, relative stability of composition and physical and chemical properties of the internal medium after some time is disturbed and life is stopped.

Cellular homeostasis in a healthy person is determined by the balance between cell death and cell reproduction. Every day, a healthy adult has 50 - 70 billion new cells, and the same number of them die, Their place is occupied by new cells. In general, in a year, so many cells are updated in a person that their total weight is equal to the weight of the body.

The process of maintaining optimal cell numbers - cellular homeostasis - carries out a complex system of control of cell divisions (proliferation) and cell death, mainly due to apoptosis. In the process of apoptosis, the cell disappears without trace within 15-120 minutes.

Apoptosis is a programmed cell death, an energetically dependent, genetically controlled process that is triggered by specific signals and rid the body of weakened, unnecessary, or damaged cells. Apoptosis is a biological sanitizer. In a healthy body, anything that is not adapted to new conditions must necessarily be removed. Therefore, death (selfdestruction) occurs incorrectly developing, potentially dangerous or simply unnecessary for surrounding tissues cells.

\section{Objectives and objectives of the study}

The aim of the study of the cell mechanism of self-healing is to detect cellular processes of maintaining the healthy state of the organism. The aim of the study is to identify activities that support human health at the cellular level.

\section{Studies of cell self-healing of a healthy condition}

The quality state of cells depends on the state of the body. By their qualitative improvement through the system of cellular self-healing of the organism, a healthy state of cells at the biological level is achieved by charging, gymnastics and physical education, working, rest, food and sleep time, setting of life systems, healthy nutrition, clean ecology, coordination of life activity with the natural daily cycle. The main processes of self-healing of the body at the biological level are hormonal equilibrium, metabolism and regeneration. In order to normalize metabolism, it is necessary to switch to alkaline nutrition, excluding from the diet carcinogenic food obtained by chemical processing, containing mutation proteins; to drink clear water.

${ }^{*}$ Correspondence to: Evgeniy Bryndin, Research Department, Research center “NATURE INFORMATIC”, Novosibirsk, Russia, E-mail: bryndin15@yandex.ru

Key words: hormonal balance, metabolism, regeneration, healthy condition, cellular level, healthy lifestyle

Received: February 17, 2020; Accepted: February 25, 2020; Published: February 28,2020 
In normal metabolism, hormonal equilibrium requires coordination of vital activity with the natural daily cycle. When the natural rhythm of sleep and waking is disrupted, then the hormone melatonin is not sufficiently produced, the hormonal balance in the body is disturbed and, as a result, the mechanism of cellular renewal is disrupted, and cells continue to grow or degenerate. In order to begin the process of death of reborn cells, it is necessary to suspend the action of their mutation genes by genetic inductors (hormones). This is achieved by hormonal equilibrium on hormone production and normalization of the hormonal background on delivery to their cells. In order to establish hormonal balance, it is necessary to produce melatonin hormone by epiphysis in sufficient amount. In daylight, melatonin is not produced. Melatonin is produced at night during sleep. For this purpose it is necessary to first establish a sleep regime in order to produce the hormone melatonin in sufficient quantity. For the full production of hormones by the hormonal system it is necessary to go to nature after sleep to coordinate the internal and external environment through the adaptation system in order to produce neurotransmitters hypothalamus, then before breakfast consuming blueberries to maintain hormonal balance and before dining sea buckthorn to normalize hormonal background.

Cells are regenerated during their division through metabolism, normal metabolism and hormonal equilibrium. Regeneration of vertebral disks and cartilage tissue of joints is carried out in the process of uniform walking. In uniform walking, regeneration of cartilage tissue compensates for its erasure. After 20 minutes of even walking, the feeding of vertebral cells by diffusion as well as the regeneration process begins. Complete regeneration of vertebral disks is carried out in 1.5-2 hours of uniform walking, 64 steps per minute.

In order to normalize the mechanism of cellular renewal (regeneration), it is necessary to adjust daily life systems and holistic functioning of the body.

In order to maintain and form a healthy state through the system of cellular self-healing of the organism, it is necessary to:

1. Provide the vibration frequency of cells 8 hertz long 8 meters with a holistic setting of the body.

2. Normalize the body rhythms through the system of internal breathing by uniform walking 64 steps per minute with combined (abdominal, thoracic, laryngeal and airy sinuses) breathing by 4 steps of breath and 4 steps of exhalation through the nose to maintain resonance of frequencies of healthy cells by their complete interference.

3. Maintain at the energy level the resonance of the frequencies of healthy cells by the current of the earth 's nucleus, which passes through the energy channel of the spine, the elimination of energy plugs in the energy channels and the holistic adjustment of the body 's energy system.

4. Maintain the regeneration of healthy cells with a healthy spirit, healthy nutrition, metabolism, normalization of the rhythms of body functioning, adjustment of life systems, holistic adjustment of the body, life activity in a favorable natural and social environment in summer, autumn, winter and spring.

5. It is necessary to form the quality of spirit from conception. Spirit in every cell. Body without spirit dead.

6. Energy normalization of cell resonance with earth core current. Energy waves affect the wave gene - the wave gene triggers the genetic system - the genetic system triggers the hormonal system. Hormones are genetic inducers to turn genes and mutagens on and off.

7. Physiological normalization of cell resonance by body rhythms. Healthy nutrition, clean natural and social environment, physical exercises, day regime, coordination of social life with natural rhythms physiologically normalizes the state of the body.

8. Anatomical normalization of the body 's cellular self-healing system. The correct poses during sleep, work, rest and food normalize the anatomy of the body.

Human life is defined by physiological reserves. Physiological reserves are limit values of body life systems, such as body temperature, arterial and venous pressure, composition and physical-chemical and biological properties of blood, acid-alkaline balance of all biological media and others. Reserve living conditions of a person show that he can cost 40 days without food, 12 days without sleep, 6 days without water, 5 minutes without air, body temperature can be not less than 25 degrees and not more than 41 degrees.

One mechanism that increases life expectancy is autophagia (self-eating of cellular debris - incorrectly folded proteins, damaged mitochondria, etc.). In this process, damaged components of the cell are delivered inside its lysosomes, where they undergo digestion

Accumulation of cellular debris harms the whole body contributes to accelerated aging and development of diseases, reduces life expectancy.

Under normal living conditions, cells have no need to eat than they will have to and use any unnecessary sludge. Autophagy is only triggered when certain conditions occur, namely nutrient deficiency.

In autophagy, cells are purified, rejuvenated, and become more viable. Therefore for prevention of aging and the diseases associated with it, it is necessary from time to time to force an organism to hold such community work days on cleaning of an organism, starting an autofagiya.

Autophagy is the adaptable response of a cell to survive adverse conditions. In autophagy, old cells are restored and new cell structures are formed.

With abundant food resources, the protein is mTOR active, and there are processes of building new proteins in the body. Suppression of mTOR stops the synthesis of new proteins and forces the restoration of old proteins. That is, mTOR inhibition triggers the autophagy process.

In order to protect against accelerated aging, autophagy must be launched from time to time in order to increase life expectancy. For this purpose it is possible to arrange unloading vegetable days (only vegetables and water) or short fasting. As well as low calorie nutrition activates autophagy.

The term autophagy means self-eating and refers to processes by which your body cleans up various debris, including toxins and regenerates damaged cell components.

"Your cells create shells that hunt for pieces of dead, sick or worn out cells; devour them; smooth out them; And use the resulting molecules for their energy or the production of new cell parts. "

By increasing autophagy in your body, you reduce inflammation, slow aging, and optimize biological functions. "More autophagy occurring in tissues should mean fewer damaged and weakened cells at any one time, which in turn should lead to a longer life of the body." 
Exercise is one way in which the level of autophagy is increased. Physical exercise creates mild damage to muscles and tissues, which causes the body to then engage in repair, and thereby make the body stronger. Exercise also helps to get rid of toxins by sweating, which is useful for detoxification. In fact, many researchers see physical load as a fundamental aspect of effective detoxification. Dr. George U., for example, who has taken part in clinical detoxification trials, recommends using a combination of exercise, sauna and supplements with niacin to increase the excretion of toxins through the skin.

Studies show that the ideal area in which exercise shows the greatest benefit to increase life expectancy ranges from 150 to 450 minutes of moderate exercise per week.

Limit your protein intake to about 40-70 grams per day, depending on your muscle body mass. The best formula is one gram of protein per kilogram of muscle body mass

Healthy mitochondria are the basis for preserving your health and preventing disease. Mitochondrial lesions can cause genetic mutations,

Autophagia is one way to remove damaged mitochondria, and biogenesis is the process by which new healthy mitochondria can be duplicated. Exercise plays a twofold role because it not only stimulates autophagy processes, but is also one of the most powerful stimulators of mitochondrial biogenesis. By stimulating your mitochondria organelles located in almost every cell that produce ATP, you allow mitochondria to start creating active oxygen forms (APCs) that act as signaling molecules. One function of this signal is to stimulate the production of more mitochondria. In fact, the key to preventing disease, which practically excludes the risk of developing cancer, heart disease, diabetes, many other diseases and slowing down aging processes, is to optimize mitochondrial functions and increase the number of these mitochondria. Fortunately, exercise helps make these two useful things.

Nutritional restriction is another biological stressor that produces many beneficial results, including enhancing autophagy. In fact, some of the benefits associated with nutritional restriction are known: reducing the risk of developing diabetes and heart disease.

Meals between 8 a.m. and 4 p.m. can be a much better schedule for some people, and this schedule has the added advantage because it allows you to fast for hours before bed. Dr Mercola is convinced this is the best choice for most people not to eat in the three hours before bed.

There is compelling evidence showing that when supplying fuel for your mitochondria at a time when they don ' $t$ need it, leads to the leakage of a large number of electrons that release active oxygen forms, acting as free radicals. These free radicals damage mitochondrial, and ultimately nuclear DNA. It is necessary to strive for starvation for six hours before bed, but if we talk about the minimum, we should not eat at least three hours before sleep.

The body 's photosynthetic cells receive energy from the environment and food. The energies of the external environment are accumulated by the body through energy centers, which convert them into a form for absorption by cells and organs. For example, the energy centers of light energy are associated with the glands of the endocrine system. The body accumulates up to $80 \%$ of the energy from the external environment necessary for the functioning of the human body. The rest of the energy is provided by food.

The human body presents an oscillating circuit. In the physical body there is a complex, combined, hierarchical, volumetric system of connected standing waves of different quality: electric, magnetic, thermal, light, gravitational, mental. Cells are the source of the oscillations. Electromagnetic standing waves form a biofield.

Human health is psycho-physiological state with balanced psyche and resonance of cells in the biofield at vibration frequency of 8 hertz [1].

How many healthy years we will have determines our way of life. It is necessary to move and lead a healthy lifestyle [2-8]. A healthy lifestyle forms and maintains a healthy state.

\section{Technique of transition to healthy lifestyle}

Transition to healthy lifestyle is carried out in four stages of formation of useful habits.

Stage 1. Formation of useful habits of maintenance of hygienic and endoecological purity of an organism. Habits are formed on performance of hygiene and endoecology. The clean environment of an organism and cages is one of necessary conditions of a wave resonance of cages. Purity - guarantee of health.

Stage 2. Development of useful habits of achievement of spiritual, and power purity and healthy state. Spiritual and power purity are necessary conditions of a wave resonance of cages.

Stage 3. Acquisition of useful habits health of saving for preservation of a healthy state.

Stage 4. Accumulation of experience of preservation of a healthy state useful habits within a year. Accumulation of experience is carried out by useful habits health of saving in various house, social and natural seasonal conditions (in the spring, in the summer, in the fall and in the winter).

\section{Biogenic health maintenance in ontogenesis}

The leading correlations in ontogenesis are genomic. They are the basis of other correlations whose significance changes throughout ontogenesis. This is due to the primacy of genotype changes in the phylogenesis process. Genomic correlations are ensured by the integrity of the genetic constitution of a developing organism.

The mechanisms of genomic correlations are genotype gene balance, concatenated gene inheritance, different forms of gene interaction, and pleiotropy.

The correlation system itself undergoes transformations. More partial correlations are modified initially, while the most general correlations can be reproduced in particular ontogenesis for a very long time. As a result, both the selection of the most general correlations, which are important in any restructuring of the body and in different living conditions (general design plan, correspondence between the degree of development of the nerve center and innervated organs), as well as the accumulation of local correlations of private importance, which arise in the body and reflect the specifics of their lifestyle.

Correlations of general importance cause continuity of formative processes of the organism of this type of organization, and private correlations - diversity of specific forms of life.

There is a close link between phylogenetic coordinates and ontogenetic correlations

A comparison of specific forms of organ correlation transformations leads to the conclusion that morphogenetic correlations and topographic co-ordinations, just like ergontic correlations and 
dynamic co-ordinations, are mutually determinative. Morphogenetic correlations occur between organs spatially related to each other. Ergontic correlations are due to the functional interdependence of organs and parts of the body.

In the pair, genomic correlations - biological coordinates are also found to match. The relative transformation of biological structures is at the heart of the normal development of the human body. The mechanisms of relative transformations of biological structures in phylogeny are different, and therefore three groups are identified: biological, dynamic and topographic. Biological co-ordination is observed between structures not directly related in function or location. The main link between them is adaptations to certain habitat conditions. Dynamic coordination is expressed in the mutual correspondence of structures linked functionally. Topographic coordination manifests itself between structures connected to each other spatially. All types of coordination are highly stable.

Biogenic correlations are carried out at the stage of body formation up to 22 years, body aging and body state formation (healthy, donosological, patient) during life. The genetic apparatus works on two levels: real and wave. The real level responsible for protein synthesis works on a discrete principle. The wave level works on an analog principle. The accumulated instrumental mental energy affects the human genome at the wave level. Genetic apparatus converts wave analogue signal of psychic energy into discrete signals of biogenic correlation of psychophysical processes and body state. It is important for a person to accumulate the instrumental mental energy of healthy life [9-15]. Then biogenic correlations will maintain a healthy state in any natural and social conditions year after year during life.

\section{Conclusion}

The technique of achieving and maintaining health at the cellular level determines a healthy state; Useful actions to achieve a healthy state; Acquisition of useful habits by emotional consolidation of useful actions; Qualitative complex improvement of cells state at spiritual, energetic, physiological and anatomical levels in social, natural and home conditions for achieving healthy state by useful actions through the system of cellular self-healing of the organism at the stage of formation of health-improving abilities; Qualitative complex preservation of cell state at spiritual, energy, physiological and anatomical levels in social, natural and home conditions for achieving healthy state by useful habits through the system of cellular self-healing of the organism at the stages of acquisition of health saving skills and accumulation of mental energy of healthy lifestyle. Hygiene and endoecology, light bioenergy and natural ecology, balanced mentality and healthy lifestyle are criterion of maintenance of healthy state [4].

\section{References}

1. Bryndin EG, Bryndina IE (2018) Natural technology of high quality transition to healthy activity. Int $J$ Health Sci Res 3: 24-32.

2. Bryndin E, Bryndina I (2018) Natural and spiritual aspects of health and social infrastructure of public health care on base of healthy lifestyle. Medical Open Journal Gerontology \& Geriatrics 3: 404-408.

3. Evgeniy B, Irina B (2018) Adaptive Ecological Social Technology of High-Quality Transition to Healthy Long Activity. Res \& Rev Health Care Open Acc J 3: 226-232.

4. Bryndin E, Bryndina I (2019) Hygiene and endoecology, light bioenergy and natura ecology, balanced mentality and spiritual life as criterion of health. JMRHS 2: 543-551.

5. Bryndin E, Bryndina I (2019) Natural science approach to determination of health and formation of healthy lifestyle. ASMS 3: 26-37.

6. Bryndin E, Bryndina I (2019) Development of health care on basis of healthy lifestyle for forming future medicine of longevity. ASMS 3: 35-41.

7. Bryndin E, Bryndina I (2019) International public health care on basis healthy lifestyle. Asian Journal of Medical Science Research \& Review 1: 88-96.

8. Bryndin E, Bryndina I (2019) Training of Healthy Lifestyle in Educational Schools Asclepius Medical Case Reports 2: 1-3.

9. Bryndin EG, Bryndina IE (2016) Normalization of cognitive thinking by healthy lifestyle. ARC Journal of Public Health and Community Medicine 1: 1-6.

10. Bryndin EG, Bryndina IE (2019) Normalization of psyche by healthy lifestyle of various groups of the population. jmcrr 2 .

11. Bryndin EG (2018) Psychological and social aspects formations of thinking, consciousness and behavior. SM Physical Medicine \& Rehabilitation 2: 1-5.

12. Bryndin E (2018) Satisfaction with valuable realization of requirements by spiritual personality counterbalances psyche. MOJ Public Health 7: 254-257.

13. Bryndin E, Bryndina I (2019) Health Maintenance by Balanced Psyche, Spiritual Life, Hygiene, Endoecology and Ecological Nature. Research and Reviews on Healthcare: Open Access Journal 4: 322-328.

14. Bryndin E, Bryndina I (2019) Supporting technology of vigorous activity by normalization of biofield and by healthy lifestyle. Int J Healthc Med Sci 5: 1-11.

15. Bryndin E, Bryndina I (2019) Normalization of Psyche of the Motivated Population by Healthy Lifestyle. Lupine Online Journal Medical Sciences 4: 364-367.

Copyright: (C2020 Bryndin E. This is an open-access article distributed under the terms of the Creative Commons Attribution License, which permits unrestricted use, distribution, and reproduction in any medium, provided the original author and source are credited. 